\title{
EDITORIAL
}

\section{The Sarnat score for neonatal encephalopathy: looking back and moving forward}

\author{
Pediatric Research (2020) 88:824-825; https://doi.org/10.1038/s41390- \\ 020-01143-5
}

In 1976, Sarnat and Sarnat ${ }^{1}$ published a study of 21 neonates with encephalopathy attributed to a "well-defined episode of fetal distress or an Apgar score of $\leq 5$ at 1 or 5 min after delivery." Their staging system for the sequential evolution of clinical signs and electroencephalogram (EEG) changes was intended to facilitate formulation of prognosis for neurologic outcome. ${ }^{1}$ This descriptive study has endured over the decades, because it was the first of its kind to propose a systematic approach to the common problem of neonatal encephalopathy, and because it is easily administered at the bedside. Modifications of the Sarnat Scoring System have been employed in the major trials of therapeutic hypothermia for neonatal hypoxic-ischemic encephalopathy (HIE) to identify neonates at highest risk for abnormal neurodevelopmental outcome. ${ }^{2}$ Today, 44 years later, the Sarnat score is a widely used tool worldwide to identify term neonates with encephalopathy, and particularly those who qualify for therapeutic hypothermia. Here we revisit the original article by Sarnat and Sarnat, ${ }^{1}$ including its purpose and limitations, present applications, and the need for ongoing adaptation and study.

In their original study, Harvey B. Sarnat and Margaret S. Sarnat delineated degrees of encephalopathy for term and near-term neonates following fetal distress. While their scale was not intended to define diagnostic criteria for HIE, the series included neonates who had suffered presumed perinatal hypoxia and/or ischemia. Infants were described by the duration spent in each of three stages of encephalopathy based on clinical exam findings and EEG features. In stage 1, infants exhibited hyperalertness, hyperreflexia, sympathetic autonomic responses, and a normal EEG. When present, stage 1 typically lasted from 1 to $18 \mathrm{~h}$. In stage 2, infants displayed obtundation, mild hypotonia, flexor posturing, parasympathetic responses, and seizures. Stage 2 in some cases followed stage 1, while in others was present from birth, on average lasting about 5 days. Finally, in stage 3 infants were stuporous, flaccid, had absent or suppressed brainstem and/or autonomic function, and had either fully attenuated or abnormally discontinuous EEGs. Stage 3 was not present in all subjects, but those who demonstrated stage 3 findings had worse outcomes. Similarly, infants with a duration of stage 2 lasting $>5$ days had worse outcomes.

While the original Sarnat publication provides a useful clinical description of neonatal encephalopathy, it must be considered with several caveats. The first is the small number of patients included in the development of the scoring system, raising the question of generalizability. Further, since the inclusion criteria were "a well-defined episode of fetal distress" or "an Apgar score of $\leq 5$ at 1 or $5 \mathrm{~min}$ after delivery," the underlying pathogenic mechanisms could be heterogeneous. Indeed, the authors explicitly state, "None of the clinical or electroencephalographic characteristics here described are specific for postanoxic encephalopathy, but rather are manifestations of a state of functional impairment of the brain." As originally described, the Sarnat scale emphasized the dynamic nature of encephalopathy. All 21 of the neonates described in the original paper had evolution of their exams and passed through stage 2 at some point in their course, highlighting the idea that timing of scoring is crucial. In contrast, as typically applied in recent years, the Sarnat score provides only a single snapshot to identify neonates with encephalopathy in the first $6 \mathrm{~h}$ of life, limiting its scope as both a diagnostic and prognostic tool. Finally, the authors suggested that infants who did not reach stage 3 and infants who recovered from stage 2 within 5 days had good neurodevelopmental outcomes by 1 year. However, with such a small number of subjects, it is difficult to draw firm prognostic conclusions that can be generalized to clinical care. In addition, there was never validation of these findings in a separate cohort.

To address some of these limitations, subsequent work sought to refine characterization of neonatal encephalopathy. In 1997, Thompson et al. $^{3}$ tested a numeric scoring system with fewer clinical assessment-based items in 45 neonates with HIE. In contrast to the Sarnat scale, the Thompson score did not require categorization of severity of encephalopathy but rather relied on a simple numeric score to describe the peak severity of encephalopathy. By design, the Thomspon score did not require specific training or depend on the availability of advanced technologies (e.g., magnetic resonance imaging, computed tomography, cerebral function monitoring). The score consisted of clinical assessment of nine signs: tone, level of consciousness, seizures, posture, Moro, grasp, suck, respiratory pattern, and fontanelle findings. Each sign was scored from 0 to 3 (maximum score of 22, reflecting greatest severity) and was performed daily until a score of 0 was achieved or the infant was discharged from the hospital. The study found that a maximum score of $>10$ during the first 7 days was highly predictive of abnormal outcome at 12 months of age with $100 \%$ sensitivity and $61 \%$ specificity. However, this system also had a number of limitations, including unclear inclusion criteria for infants ("if clinical signs of hypoxic-ischemic encephalopathy developed after birth"), as well as a small sample size. Only a single score was assigned per day, with the peak score from any time in the first 7 days used for prognostication. This is of particular importance since it does not reflect use of the scoring system to determine whether an infant should undergo therapeutic hypothermia within $6 \mathrm{~h}$ of birth. Nonetheless, there remains value in the Thompson score as a diagnostic and predictive tool; further work is warranted to clarify its validity in the initial hours of life and how it might best be used in combination with ancillary studies, such as EEG and imaging, to inform prognosis.

Another simple encephalopathy score was proposed by Miller et al. ${ }^{4}$ in 2004 based on assessments of feeding, alertness, tone, respiratory status, reflexes, and seizure activity. Each was scored as either normal $(=0)$ or abnormal ( $=1$ point for each category). The score was assigned once daily, with a maximum score of 6 , reflecting greatest severity. The score on the first day of life and the maximum score within the first 3 days of life were used for 
analysis. The goal was to validate this simple and quantifiable encephalopathy score for predicting 30-month neurodevelopmental outcomes. The authors found that within the first 3 days of life this score identified the newborns at highest risk of abnormal outcome, at a time that was still relevant for acute clinical decision-making. Nevertheless, this system also has its limitations, such as not being able to distinguish among the heterogeneous causes of neonatal encephalopathy and no longitudinal analysis of how the score correlates with evolution of the exam over time. Lastly, both the Thompson ${ }^{3}$ and Miller ${ }^{4}$ scores included the presence of clinically evident seizures as a criterion but omit electrographic information. Clinical diagnosis of neonatal seizures is unreliable and EEG confirmation of paroxysmal episodes in newborns is a crucial diagnostic tool. Subsequent models, such as that of Ambalavanan et al., ${ }^{5}$ have proposed the combination of elements of the Sarnat scale with additional data, such as laboratory data, or other clinical information in order to improve the prognostic accuracy. While these may be informative, they do not address the challenge of accurate, early identification of HIE.

The original paper by Sarnat and Sarnat ${ }^{1}$ laid the invaluable groundwork for our current understanding of the evolution and degrees of neonatal encephalopathy. Even so, further investigation is needed to synthesize decades of cumulative experience into a simple, validated screening tool that can be used at the bedside to expediently diagnose the severity of neonatal encephalopathy and provide specific prognostic information. The role of early ancillary tests to aid the diagnosis of HIE will be a fundamental component of future study. When available, EEG data ought to be incorporated to guide providers' discussions around management and outcomes.

This area should remain a research priority in the Neonatology and Neurology communities. While promising discoveries in neuroprotective therapies continue, these will only be as useful as our diagnostic tools allow to quickly and accurately identify those neonates who would benefit most from intervention. There is an urgent need for ongoing research to develop and validate clinical screening tools to identify HIE that may be easily applied across a variety of settings in the first hours of life and provide reliable information about the spectrum of severity.

\section{ACKNOWLEDGEMENTS}

C.J.W. is supported by K02NS102598.

\section{AUTHOR CONTRIBUTIONS}

Substantial contributions to conception and design, drafting the article or revising it critically for important content, and final approval of the version to be published-all authors.

\section{ADDITIONAL INFORMATION}

Competing interests: The authors declare no competing interests.

Patient consent: Patient consent was not required for this paper.

Publisher's note Springer Nature remains neutral with regard to jurisdictional claims in published maps and institutional affiliations.

Anna Mrelashvili ${ }^{1}$, Jeffrey B. Russ ${ }^{1,2}$, Donna M. Ferriero ${ }^{1,2,3}$ and Courtney J. Wusthoff (iD)

${ }^{1}$ Department of Pediatrics, University of California San Francisco, San Francisco, CA, USA; ${ }^{2}$ Department of Neurology, University of California San Francisco, San Francisco, CA, USA; ${ }^{3}$ Weill Institute for Neurosciences, University of California San Francisco, San Francisco, $C A$, USA and ${ }^{4}$ Departments of Neurology and Pediatrics, Stanford University, Stanford, CA, USA

These authors contributed equally: Anna Mrelashvili, Jeffrey B. Russ. Correspondence: Courtney J. Wusthoff (wusthoff@stanford.edu)

\section{REFERENCES}

1. Sarnat, H. B. \& Sarnat, M. S. Neonatal encephalopathy following fetal distress. A clinical and electroencephalographic study. Arch. Neurol. 33, 696-705 (1976).

2. Jacobs, S. E. et al. Cooling for newborns with hypoxic ischaemic encephalopathy. Cochrane Database Syst. Rev. 2013, CD003311 (2013).

3. Thompson, C. M. et al. The value of a scoring system for hypoxic ischaemic encephalopathy in predicting neurodevelopmental outcome. Acta Paediatr. 86, 757-761 (1997).

4. Miller, S. P. et al. Clinical signs predict 30-month neurodevelopmental outcome after neonatal encephalopathy. Am. J. Obstet. Gynecol. 190, 93-99 (2004).

5. Ambalavanan, N. et al. Predicting outcomes of neonates diagnosed with hypoxemic-ischemic encephalopathy. Pediatrics 118, 2084-2093 (2006). 